\title{
Prediction of in-hospital mortality and admission to ICU using vital signs - a study of Early Warning Score as an alternative to traditional triage
}

\author{
Momo Menna Illum Vendler', Tobias Thostrup Andersen ${ }^{1 *}$, Charlotte Barfod ${ }^{2}$, Jakob Lundager Forberg ${ }^{2}$ \\ From Proceedings of the 5th Danish Emergency Medicine Conference \\ Aarhus, Denmark. 18-19 April 2013
}

\section{Background}

Triage of patients in the Emergency Department includes scoring of vital parameters. The objective of this study was to compare two such triage systems for assessing vital parameters - a single-parameter system, T-vital, as used in Danish Emergency Process Triage, and a multiple-parameter system, T-EWS, which we based on Early Warning Score (EWS) - and correlate the triage scores to in-hospital mortality and admission to ICU. Studies examining EWS in triage are currently limited in number.

\section{Methods}

Using data from the Acute Admission Database of Nordsjællands Hospital ( $\mathrm{n}=6164$ admissions), we calculated and stratified EWS into four T-EWS colour codes (red, orange, yellow, and green), testing different stratifications' correlation to in-hospital mortality and admission to ICU. Afterwards, we compared the ability of the chosen T-EWS and T-vital to predict patients at risk (red and orange category) of in-hospital mortality or admission to ICU. The data were analysed using area under the receiver operating curve (AUROC), sensitivity, specificity, overtriage, undertriage, and diagnostic rates.

\section{Results}

T-vital allocated $10.6 \%$ of patients to the orange or red category, whereas T-EWS allocated $5.8 \%$ to these categories. There was no significant difference in the ability of T-EWS to predict in-hospital mortality compared to

* Correspondence: tobias.ta@gmail.com

${ }^{1}$ University of Copenhagen, Denmark

Full list of author information is available at the end of the article
T-vital (AUROC $(95 \% \mathrm{CI})$ : T-EWS $=0.74(0.70-0.79)$; $\mathrm{T}$-vital $=0.76(0.72-0.80))$. Likewise, there was no significant difference in prediction of ICU admission (AUROC (95\% CI): T-EWS $=0.76(0.70-0.81)$; T-vital $=0.73(0.67$ $0.79)$ ). The specificity $(95 \% \mathrm{CI})$ of T-EWS compared to $\mathrm{T}$-vital was higher for both in-hospital mortality $(0.95$ (0.94-0.95) and 0.90 (0.90-0.91), respectively) and for admission to ICU (0.95 (0.94-0.95) and 0.90 (0.89-0.91), respectively). There was a trend of higher sensitivity of $\mathrm{T}$-vital, and no difference in overtriage, undertriage or diagnostic rates.

\section{Conclusion}

The two triage systems are largely similar in their ability to discriminate patients at high risk of in-hospital mortality or admission to ICU. However, T-vital's larger proportion of orange and red patients might yield a larger workload in the Emergency Department. Replacement of T-vital with T-EWS could be considered, as EWS is already in use as a monitoring tool after triage, but more studies are needed for further clarification.

\section{Authors' details \\ ${ }^{1}$ University of Copenhagen, Denmark. ${ }^{2}$ Emergency Department, Nordsjæellands Hospital, Denmark.}

Published: 9 September 2013

doi:10.1186/1757-7241-21-S2-A40

Cite this article as: Illum Vendler et al.: Prediction of in-hospital mortality and admission to ICU using vital signs - a study of Early Warning Score as an alternative to traditional triage. Scandinavian Journal of Trauma, Resuscitation and Emergency Medicine 2013 21(Suppl 2):A40.

\section{() Biomed Central}

(c) 2013 Illum Vendler et al; licensee BioMed Central Ltd. This is an Open Access article distributed under the terms of the Creative Commons Attribution License (http://creativecommons.org/licenses/by/2.0), which permits unrestricted use, distribution, and reproduction in any medium, provided the original work is properly cited. 